Neurosurg Focus 13 (3):Article 3, 2002, Click here to return to Table of Contents

\title{
Surgical management of unruptured basilar artery bifurcation aneurysms
}

\author{
Technical note
}

\author{
Aaron S. Dumont, M.D., Rod J. Oskouian, Jr., M.D., Michael M. Chow, M.D., \\ AND NEAL F. KASSELL, M.D. \\ Department of Neurological Surgery, University of Virginia Health Sciences Center, Charlottesville, \\ Virginia
}

\begin{abstract}
The basilar artery (BA) bifurcation is the most common site for aneurysms arising from the posterior circulation. Their inhospitable location, nested within the narrow confines of the interpeduncular fossa anterior to the brainstem, coupled with the rich network of adjacent critical thalamoperforating arteries irrigating the midbrain and thalamus, pose difficult anatomical obstacles for the surgeon.

The age old adage that the only cure for intracranial aneurysms remains exclusion from circulation before rupture still holds true. Although management of unruptured aneurysms in general is still controversial, unruptured aneurysms of the BA bifurcation can be treated surgically with acceptable rates of morbidity. The clinician must gather and weigh all clinical, pathological, and radiological data when formulating recommendations for the individual patient.

In the present report the authors describe their current technique for the surgical management of unruptured BA bifurcation aneurysms; this represents the culmination of the senior author's (N.K.) experience in the management of both ruptured and unruptured BA bifurcation aneurysms. A modified, right-sided subtemporal transtentorial approach has been adopted in all cases of isolated unruptured BA bifurcation aneurysms. Technical nuances are described.
\end{abstract}

\section{KEY WORDS • aneurysm • basilar artery bifurcation • unruptured intracranial aneurysm • subtemporal approach}

Aneurysms arising from arteries of the posterior circulation comprise approximately 10 to $15 \%$ of all intracranial aneurysms. Posterior circulation aneurysms (or vertebrobasilar aneurysms) are, however, a formidable affliction for both patients and clinicians alike. These aneurysms, situated in the inhospitable region anterior and/or adjacent to the brainstem and cerebellum, have posed a particular therapeutic challenge. Factors including their relative rarity (allowing few surgeons to be adequately experienced in their management), the narrow confines in which they are situated, and their proximity to eloquent and unforgiving structures have impeded the development and refinement of efficacious treatment techniques that might be applied with acceptable risk. Posterior circulation aneurysms have only recently been treated with similar outcomes to those of their anterior circulation counterparts. The development and evolution of surgical management for posterior circulation aneurysms has been orchestrated by several preeminent neurosurgeons. Homage for the fact that posterior circulation an-

Abbreviations used in this paper: $\mathrm{BA}=$ basilar artery; $\mathrm{CSF}=$ cerebrospinal fluid; SCA = superior cerebellar artery. eurysms can now be treated throughout the world effectively and safely must largely be paid to Dr. Charles Drake and his disciples for their seminal contributions. ${ }^{1-5}$

The BA bifurcation remains the most common site for posterior circulation aneurysms. Arguably, aneurysms arising from the BA bifurcation remain the most difficult of all aneurysms to treat surgically because of their location deep within or near the interpeduncular fossa and because of the rich network of critical perforating arteries in the vicinity that irrigate the brainstem and thalamus.

We have noted an increased number of referrals for patients harboring unruptured aneurysms. This is probably due to relatively recent publicity devoted to unruptured intracranial aneurysms, increased vigilance of the referring physicians, and the emergence and widespread availability of noninvasive neuroimaging techniques. Every patient is counseled individually, but each is informed of the management options (including conservative, surgical, or endovascular strategies [or a combination thereof]) in detail.

Presently, surgical management of unruptured BA bifurcation aneurysms at the University of Virginia is reserved for patients in whom the presence of comorbidities would preclude surgical therapy, those whose aneurysms 
have recently become symptomatic (for example, due to mass effect), those whose aneurysms have exhibited growth or geometric change on serial imaging studies, and for those whose aneurysms exhibit characteristics that may predispose to rupture (such as aneurysm wall irregularities, although this latter indication remains speculative). We have also managed entirely asymptomatic patients who adamantly want to undergo surgery regardless of risk and have honored these patients' wishes when reasonable. Age has not been a primary exclusion criterion. In general, an individual's clinical, pathological, and radiological data are considered during the formulation of treatment recommendations. Admittedly, counseling the patient who harbors an unruptured aneurysm remains an inexact science, and it is hoped that data accrued from future studies will facilitate the process. ${ }^{6}$

We presently use a modified right-sided subtemporal approach to all unruptured BA bifurcation aneurysms except those in patients harboring simultaneous anterior circulation aneurysms. In such cases, a "half-and-half" approach is used.

In the present paper we describe a surgical approach to unruptured BA bifurcation aneurysms, which represents the culmination of the senior author's (N.K.'s) experience in the treatment of both ruptured and unruptured BA bifurcation aneurysms. Technical nuances we found useful are described.

\section{MODIFIED SUBTEMPORAL TRANSTENTORIAL APPROACH}

Drake pioneered and perfected the subtemporal approach for aneurysms of the distal BA and posterior cerebral artery. ${ }^{1-5}$ The subtemporal approach provides excellent exposure of the interpeduncular fossa and permits proximal control of the BA irrespective of the height of its bifurcation from a lateral perspective (Fig. 1 upper left). It is perhaps ideal for low-riding BA bifurcation aneurysms and those that project posteriorly, and it may also be used in any BA aneurysm in which proximal control is difficult. The main limitation of this approach lies in its limited visualization of the contralateral $\mathrm{P}_{1}$ segment, particularly with an anteriorly projecting aneurysm sac. We have used this approach with modifications in all patients harboring isolated, unruptured BA bifurcation aneurysms.

Several adjunctive measures are undertaken during surgical treatment of these aneurysms. Surgery is performed after induction of isoflurane anesthesia. To facilitate dissection and clipping of the aneurysm, temporary clipping of the BA trunk has become routine as a measure to slacken the aneurysm. Alternatively, systemic hypotension has been induced by deepening the level of isoflurane anesthesia. To facilitate brain retraction, hyperventilation to an arterial $\mathrm{PCO}_{2}$ of 20 to $25 \mathrm{~mm} \mathrm{Hg}$ is used, a lumbar subarachnoid catheter is placed for CSF drainage, and mannitol 20\% solution (approximately $3 \mathrm{gm} / \mathrm{kg}$ ) in divided infusions is administered-half at induction and half at the time of craniotomy. Hypothermia (to a mean temperature of $34.5^{\circ} \mathrm{C}$ ) is also used in all cases.

The patient is placed on the operating room table in a supine position with the head turned $90^{\circ}$ after a shoulder roll is placed under the ipsilateral shoulder to maintain

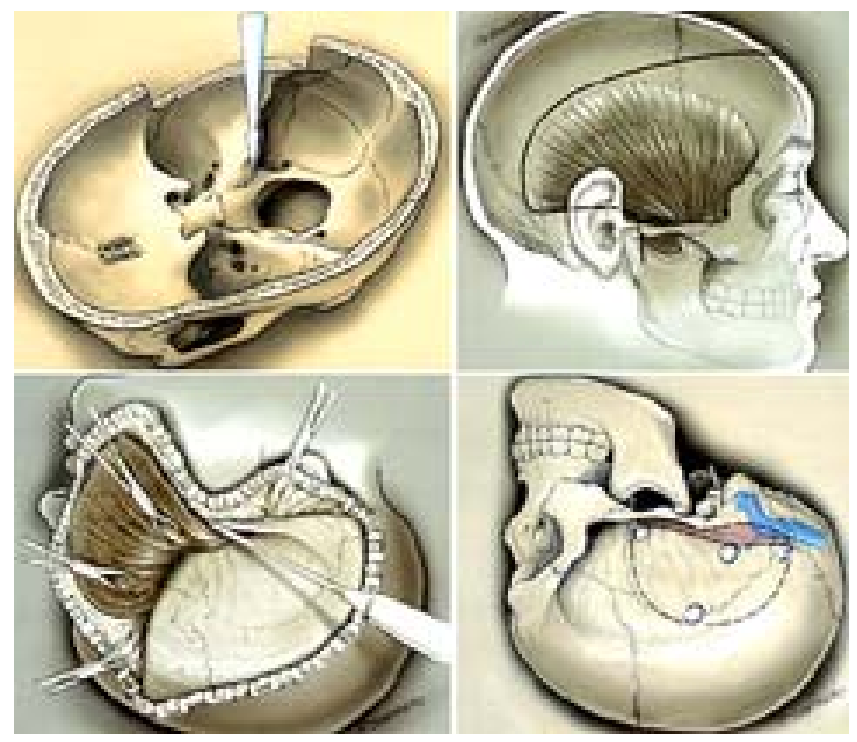

Fig. 1. Artist's illustrations. Upper Left: Corridor of access to the interpeduncular fossa. With the craniotomy performed the trajectory is a lateral one across the floor of the middle fossa. Upper Right: Planned skin incision extending from the zygoma to the asterion and above the superior temporal line, avoiding cutting of temporalis muscle fibers. Lower Left: Reflection of the skin flap as a single myocutaneous unit. Four towel clips attached with rubber bands to an outstretched elastic stocking provide retraction. Lower Right: The planned craniotomy. The burr holes are at the root of the zygoma, just above the asterion, and at the superior temporal line. The fourth and optional one lies along the floor of the middle fossa.

venous return in the neck. As mentioned, virtually all BA bifurcation aneurysms are approached from the right side. The head is placed in three-point fixation Mayfield-Kees apparatus and is shaved accordingly. The head is tilted approximately $20^{\circ}$ inferiorly to aid in retraction of the temporal lobe (and division of the tentorium).

The skin incision is begun from a point at the root of the zygoma, 1 to $1.5 \mathrm{~cm}$ anterior to the tragus (Fig. 1 upper right). The incision is extended superiorly and posteriorly to the asterion to avoid cutting temporalis fibers unnecessarily before curving superiorly and anteriorly $1 \mathrm{~cm}$ above the superior temporal line to end at a point behind the hairline. If the superior temporal artery and its branches may be encountered, they may be coagulated and divided unless necessary for a bypass procedure. Raney clips provide control of scalp bleeding. Periosteal elevators are used to separate the temporalis muscle from the bone, and the muscle is left attached to the scalp flap, wrapped in a wet lap sponge, and retracted inferiorly with the scalp flap by using three or four towel clips and rubber bands (Fig. 1 lower left). Care is taken to avoid injury to the external auditory canal while dissecting with the periosteal elevators. At this point, the lumbar drain is opened to slacken the brain.

A medium-sized bone flap is required for adequate exposure of the BA apex. The first burr hole is placed at the root of the zygoma. The superior burr hole is placed near the superior temporal line. The posterior burr hole is placed above the asterion flush with the floor of the mid- 
dle fossa, and superior and posterior to the point of insertion of the vein of Labbé into the transverse venous sinus (Fig. 1 lower right). The dura mater is stripped from the inner surface of the skull around these openings by using a right-angle dural separator and then a Penfield No. 3 dissector. Subsequently, the craniotomy is turned with a craniotome. The dura is separated from the bone subtemporally, and all bone is removed using a No. 5 cutting burr to a level flush with the floor of the middle fossa. Any opened mastoid air cells are sealed with bone wax. Epidural tack-up sutures are placed.

An inverted V-shaped dural incision is constructed. The dural opening should extend anteriorly and inferiorly to the floor of the middle fossa and posteriorly to the transverse sinus. The dura is reflected inferiorly and secured using retraction sutures. Once the brain is exposed, the vein of Labbé is inspected to allow gentle retraction of the temporal lobe away from the middle fossa floor. Every attempt is made to protect the vein of Labbé during temporal lobe retraction. The edge of the tentorium is visualized before proceeding (Fig. 2 upper left). To visualize the tentorial incisura, further temporal lobe retraction is performed. Any bleeding from the vein of Labbé is controlled by wrapping with Oxycel. Coagulation to arrest bleeding is performed only as a last resort, because venous infarction will likely result. The temporal lobe is covered with Biocol before proceeding, and the operating microscope is introduced to the field.

With due care to avoid placing traction on the third and fourth nerve, a long Greenberg retractor arm with a 5/8inch blade is placed on the inferior temporal lobe surface.

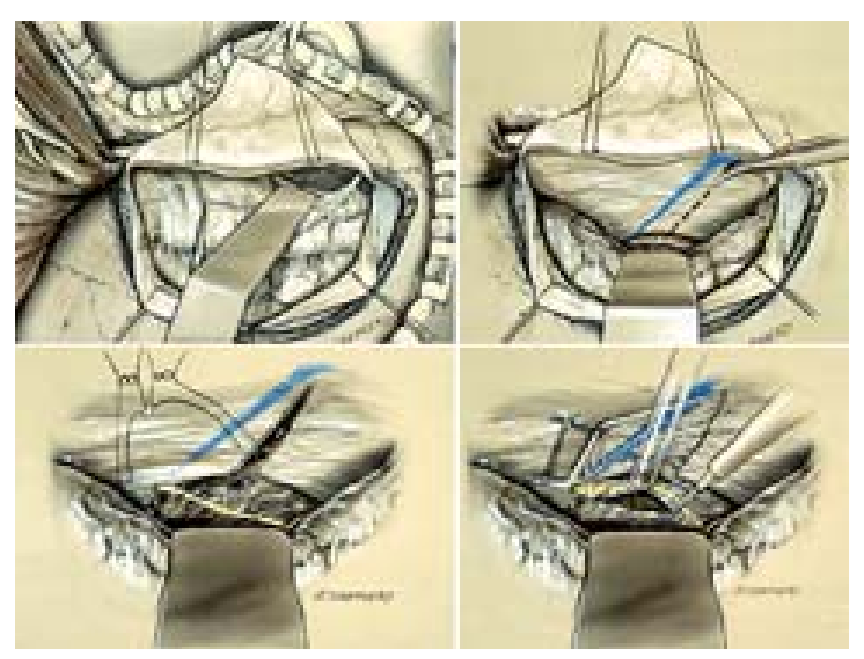

Fig. 2. Artist's illustrations. Upper Left: Dural opening and identification of the vein of Labbé. The dura mater has been opened in an inverted V shape and retracted, and the vein of Labbé has been identified at the posterior margin of the flap. Upper Right: Temporal lobe retraction and planned line of tentorial division. The temporal lobe has been carefully elevated, exposing the tentorial incisura, and the planned tentorial incision is outlined. Lower Left: Tentorial retraction. The tentorium has been divided and a suture has been placed tenting the anterior tentorial leaflet to the floor of the middle fossa. Lower Right: Opening of the ambient cistern and interpeduncular cistern. The arachnoid is being sharply opened using a beaver blade, and the fourth crainial nerve can be visualized below the tentorium.
At this point, visualization of the tentorium should be maximal. In all cases division of the tentorium is now routinely performed because it provides superior visualization of the BA and its tributaries and makes application of a temporary clip simple and fast. We have observed no complications related to this tentorial division, and it can be completed within 2 or 3 minutes. The line of the tentorial incision extends laterally from a point just below the junction of the transverse and sigmoid sinuses and medially to a point 1 to $1.5 \mathrm{~cm}$ posterior to the petrous ridge, posterior to the insertion of the fourth nerve (Fig. 2 upper right). Initially, a small incision is made laterally with a No. 11 blade, and the tentorium is meticulously coagulated using bipolar electrocautery along the proposed incision line. Next, this coagulated area is incised with the same-sized blade. This procedure is repeated (until the tentorium is divided) to ensure minimal blood loss from the tentorial sinuses and the tentorial artery of Bernasconi and Cassinari. Particular care is taken with the most medial portion of the incision where arterial bleeding is commonly encountered (Fig. 1 lower right). The anterior leaf of the incised tentorium is reflected anteriorly and laterally onto the middle fossa floor with one tacking suture. The fourth nerve should be exposed, dissected free, and protected (Fig. 2 lower left).

The arachnoid inferior to the third nerve is opened sharply, thereby providing entrance into the interpeduncular cistern and allowing further CSF drainage for brain relaxation and exposure of the SCA (Fig. 2 lower right). The SCA is tracked proximally with sharp dissection to its origin from the BA trunk, which provides access for proximal control. A portion of the BA devoid of perforators is carefully dissected and prepared for later potential planned (or emergency) temporary clipping. This is followed by dissection of the contralateral SCA and BA bifurcation. The proximal $\mathrm{P}_{1}$ segments are then identified. Exposure of the contralateral $\mathrm{P}_{1}$ segment, which is inherently difficult with this approach, may be facilitated by gentle retraction of the BA trunk posteriorly toward the interpeduncular fossa. In selected cases the aneurysm itself can sometimes be gently manipulated anteriorly or posteriorly. The aneurysm neck is then sharply dissected, and placement of the clip(s) is then undertaken. The most critical portion of this procedure remains the meticulous and absolute identification and preservation of the thalamoperforating arteries in the region. Every perforator must be carefully preserved. The simplest means of excluding the aneurysm from the circulation is chosen and is most often accomplished using a single straight or fenestrated clip. The aneurysm is then aspirated with a lumbar puncture needle to ensure obliteration and to provide further inspection of the perforators and contralateral $\mathrm{P}_{1}$ segment once the aneurysm neck is clipped.

Upon completion of clipping, the lumbar drain is closed, and $\mathrm{CO}_{2}$ and blood pressure are normalized. The dura is closed in watertight fashion. If the brain has failed to expand adequately, it is reinflated using normal saline injected via the lumbar subarachnoid catheter. The bone flap is secured with three titanium miniplates and screws, and a subgaleal/epidural Hemovac drain is placed. The temporalis muscle is tacked in place using No. 2-0 vicryl sutures in interrupted fashion, and the galea is closed using interrupted No. 3-0 vicryl sutures. The skin is approx- 
imated using a running No. 4-0 nylon suture, and a sterile head dressing is placed, which remains until the sutures are removed on postoperative Day 10.

\section{CONCLUSIONS}

Aneurysms of the BA bifurcation are the most common of those arising from the posterior circulation and probably the most difficult to treat well. Doctor Charles Drake is largely responsible for the pioneering of successful surgery for BA bifurcation aneurysms.

Although the management of unruptured aneurysms remains a matter of controversy, the contention of Drake that the only cure for intracranial aneurysms is to secure them before they rupture remains true. The pressing task of the clinician counseling such patients, however, is to select those in whom the lesion is at high risk of rupture and in whom the surgery-related risk (that is, cases in which the risk of rupture justifiably exceeds the risks of intervention). Although still inexact, treatment recommendations must be based on all available clinical, pathological, and radiological data.

We use a right-sided modified subtemporal transtentorial approach in virtually all patients with unruptured BA bifurcation aneurysms. Key points of emphasis include adjunctive measures to allow brain relaxation and protection while minimizing retraction including hyperventilation, mannitol, lumbar CSF catheter drainage, and hypothermia. Tentorial division and temporary clipping of the BA trunk are routinely used. Sharp dissection is universally used.

Potential complications include postoperative ipsilateral third cranial nerve palsies (demonstrated in $>90 \%$ of our elective procedures), pneumocephaly, CSF leakage, seizures, temporal lobe hematomas, extraaxial fluid collections, and infarctions. In the past 3 years, all patients with unruptured upper BA aneurysms have returned to their presurgical lifestyle except for two patients (one with a large BA bifurcation aneurysm and one with a giant posterior cerebral artery aneurysm) who experienced midbrain and/or thalamic postoperative infarctions.
Unruptured aneurysms of the BA bifurcation can be treated effectively and with an acceptable morbidity rate via a modified subtemporal transtentorial approach. The authors and the entire neurosurgical community, however, eagerly await the procurement of data that will confidently guide the counseling of patients harboring unruptured aneurysms. The impact of endovascular therapy will also alter the management of patients with unruptured aneurysms, and proof of its long-term efficacy is also keenly anticipated.

\section{Acknowledgment}

Support from research funds awarded by The American Heart Association was received by Dr. Dumont.

\section{References}

1. Bederson JB, Awad IA, Wiebers DO, et al: Recommendations for the management of patients with unruptured intracranial aneurysms: a statement for healthcare professionals from the stroke council of the American Heart Association. Stroke 31: 2742-2750, 2000

2. Drake CG: Bleeding aneurysms of the basilar artery: direct surgical management in four cases. J Neurosurg 18:230-238, 1961

3. Drake CG: Further experience with surgical treatment of aneurysms of the basilar artery. J Neurosurg 29:372-392, 1968

4. Drake CG: Surgical treatment of ruptured aneurysms of the basilar artery: experience with 14 cases. J Neurosurg 23: 457-473, 1965

5. Drake CG: The treatment of aneurysms of the posterior circulation. Clin Neurosurg 26:96-144, 1979

6. Drake CG, Peerless SJ, Hernesniemi JA: Surgery of Vertebrobasilar Aneurysms: London, Ontario Experience on 1767 Patients. New York: Springer, 1996

Manuscript received August 21, 2002.

Accepted in final form September 3, 2002.

Address reprint requests to: Aaron S. Dumont, M.D., Department of Neurological Surgery, University of Virginia Health Sciences Center, Box 800212, Charlottesville, Virginia 22908. email: asd2f@virginia.edu. 